University of Wollongong

Research Online

Faculty of Engineering and Information

Faculty of Engineering and Information

Sciences - Papers: Part B

Sciences

2019

\title{
High-throughput production of uniformly sized liquid metal microdroplets using submerged electrodispersion
}

\author{
Yuxin Zhang \\ University of Wollongong \\ Shiyang Tang \\ University of Wollongong, shiyang@uow.edu.au \\ Qianbin Zhao \\ University of Wollongong, qz260@uowmail.edu.au \\ Guolin Yun \\ University of Wollongong, gy417@uowmail.edu.au \\ Dan Yuan \\ University of Wollongong, dyuan@uow.edu.au
}

See next page for additional authors

Follow this and additional works at: https://ro.uow.edu.au/eispapers1

Part of the Engineering Commons, and the Science and Technology Studies Commons

Research Online is the open access institutional repository for the University of Wollongong. For further information contact the UOW Library: research-pubs@uow.edu.au 


\title{
High-throughput production of uniformly sized liquid metal microdroplets using submerged electrodispersion
}

\author{
Abstract \\ Microdroplets of gallium-based liquid metal alloys have enabled various applications in the fields of \\ biomedicine, electronics, and chemistry. However, due to the high surface tension of liquid metal, high- \\ throughput production of uniformly sized liquid metal microdroplets is challenging using conventional \\ acoustic or microfluidic methods. Here, adapting the submerged electrodispersion technique that has \\ conventionally been used for generating water-based microdroplets, we develop a simple and \\ straightforward platform for the high-throughput production of near-monodisperse (coefficient of \\ variation less than $5 \%$ ) liquid metal microdroplets in oil without using microfluidic devices. We \\ demonstrate the capabilities of this method for producing liquid metal microdroplets (diameters ranging \\ from tens to hundreds of micrometers) and introduce a spinning disk to induce a flow of oil phase for \\ preventing the coalescence of the microdroplets. The simplicity and remarkable abilities demonstrated \\ for this method may pave the path for the development of future innovative applications based on liquid \\ metal microdroplets.

\section{Disciplines} \\ Engineering | Science and Technology Studies

\section{Publication Details} \\ Zhang, Y., Tang, S., Zhao, Q., Yun, G., Yuan, D. \& Li, W. (2019). High-throughput production of uniformly \\ sized liquid metal microdroplets using submerged electrodispersion. Applied Physics Letters, 114 (15), \\ 154101-1-154101-5.
}

\section{Authors}

Yuxin Zhang, Shiyang Tang, Qianbin Zhao, Guolin Yun, Dan Yuan, and Weihua Li 


\section{High-throughput production of uniformly sized liquid metal microdroplets using submerged electrodispersion}

Cite as: Appl. Phys. Lett. 114, 154101 (2019); https://doi.org/10.1063/1.5086376

Submitted: 20 December 2018. Accepted: 01 April 2019 . Published Online: 17 April 2019

Yuxin Zhang (D), Shi-Yang Tang, Qianbin Zhao, Guolin Yun, Dan Yuan, and Weihua Li

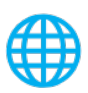

View Online

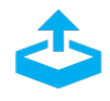

\section{ARTICLES YOU MAY BE INTERESTED IN}

Combined photo- and electroreflectance of multijunction solar cells enabled by subcell electric coupling

Applied Physics Letters 114, 153501 (2019); https://doi.org/10.1063/1.5062602

On-chip mid-infrared and THz frequency combs for spectroscopy

Applied Physics Letters 114, 150401 (2019); https://doi.org/10.1063/1.5097933

Two-wavelength thermoreflectance in steady-state thermal imaging

Applied Physics Letters 114, 151902 (2019); https://doi.org/10.1063/1.5087011

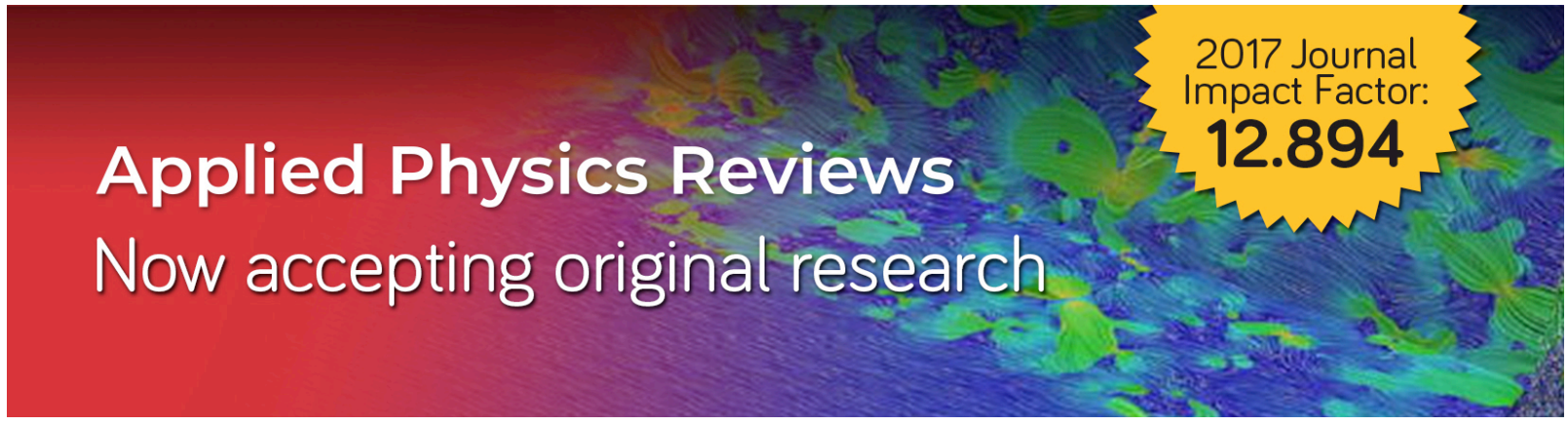




\title{
High-throughput production of uniformly sized liquid metal microdroplets using submerged electrodispersion
}

Cite as: Appl. Phys. Lett. 114, 154101 (2019); doi: 10.1063/1.5086376

Submitted: 20 December 2018 - Accepted: 1 April 2019 .

Published Online: 17 April 2019

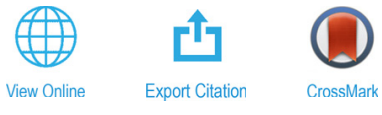

Yuxin Zhang, ${ }^{\text {a) }}$ (D) Shi-Yang Tang, ${ }^{\text {a),b) }}$ Qianbin Zhao, Guolin Yun, Dan Yuan, and Weihua Li ${ }^{\text {b) }}$

\section{AFFILIATIONS}

School of Mechanical, Materials, Mechatronic and Biomedical Engineering, University of Wollongong, Wollongong, NSW 2522, Australia

a) Contributions: Y. Zhang and S.-Y. Tang contributed equally to this work.

${ }^{\text {b) }}$ Authors to whom correspondence should be addressed: shiyang@uow.edu.au and weihuali@uow.edu.au

\begin{abstract}
Microdroplets of gallium-based liquid metal alloys have enabled various applications in the fields of biomedicine, electronics, and chemistry. However, due to the high surface tension of liquid metal, high-throughput production of uniformly sized liquid metal microdroplets is challenging using conventional acoustic or microfluidic methods. Here, adapting the submerged electrodispersion technique that has conventionally been used for generating water-based microdroplets, we develop a simple and straightforward platform for the high-throughput production of near-monodisperse (coefficient of variation less than 5\%) liquid metal microdroplets in oil without using microfluidic devices. We demonstrate the capabilities of this method for producing liquid metal microdroplets (diameters ranging from tens to hundreds of micrometers) and introduce a spinning disk to induce a flow of oil phase for preventing the coalescence of the microdroplets. The simplicity and remarkable abilities demonstrated for this method may pave the path for the development of future innovative applications based on liquid metal microdroplets.
\end{abstract}

Published under license by AIP Publishing. https://doi.org/10.1063/1.5086376

Microdroplets (diameter from few to hundreds of micrometers) of gallium-based liquid metal alloys, such as eutectic gallium-indium (EGaIn, 75 wt. \% gallium and 25 wt. \% indium) and gallium-indiumtin (Galinstan, 68.5 wt. \% gallium, 21.5 wt. \% indium, and 10 wt. \% tin), have recently been explored as the core component for realizing innovative applications in electrochemical sensing, ${ }^{1,2}$ photocatalysis, ${ }^{1,}$ constructing 3D structures, ${ }^{4}$ therapeutic bioengineering, ${ }^{5}$ flexible electronics, ${ }^{6}$ and fabricating functional composites. ${ }^{7}$ These applications are derived from the extraordinary properties of such liquid metal alloys including low melting points (below room temperature), high electrical and thermal conductivities, high deformability, being able to form a thin functional oxide layer, extremely low vapor pressure, and little or no toxicity in comparison to mercury.

Conventionally, acoustic devices such as a sonication probe system, ${ }^{3}$ a sonication bath, ${ }^{1}$ and a miniaturized piezoelectric platform ${ }^{2}$ have been used for the mass production of liquid metal microdroplets. However, droplets produced using acoustic devices are generally polydisperse with a wide distribution of sizes. Alternatively, flowfocusing microfluidic devices have been used to obtain monodisperse microdroplets of liquid metal. ${ }^{9,10}$ However, due to the high surface tension of liquid metal ( $\sim 624 \mathrm{mN} / \mathrm{m}$ for the case of EGaIn), ${ }^{11}$ the continuous phase in the flow-focusing device must be a liquid with a high viscosity to generate sufficient shear for pinching off liquid metal into discrete droplets. ${ }^{9,10}$ Nevertheless, liquid with high viscosity can induce a very high-pressure drop within the microchannels, causing deformation of the channels, tubing, and syringes that may potentially damage the system or delaminate the bonding between the microfluidic channel and the substrate. For instance, we estimate that a high pressure drop of $\sim 9.03 \mathrm{MPa}$ can be induced within a typical microchannel $(200 \mu \mathrm{m} \times 50 \mu \mathrm{m} \times 20 \mathrm{~mm})$ used for liquid metal microdroplet production when using glycerol as the continuous phase (flow rate$=25 \mu \mathrm{l} / \mathrm{min}$ ). ${ }^{10}$ Although the interfacial tension of liquid metal can be significantly reduced using electrochemistry for producing smaller microdroplets without introducing a destructive high-pressure drop, ${ }^{10}$ the throughput of the microfluidic method is still very low (flow rate of liquid metal $<20 \mu \mathrm{l} / \mathrm{min}$ ), and therefore, mass production of liquid metal microdroplets may not be feasible using microfluidic platforms. $^{9,10}$ 
Apart from acoustic and microfluidic methods, the electrodispersion technique (i.e., using electric charge to produce fine droplets from a liquid) can also be used as an alternative off-chip technique for generating controlled aqueous droplets. ${ }^{12-16}$ This method utilizes a high voltage to overcome the surface tension of a fluid meniscus at an orifice, allowing the breaking of the liquid into either monodisperse or polydisperse fine droplets (depends on the mode of operation). ${ }^{14}$ Despite the fact that electrodispersion is normally conducted in air or vacuum, it is possible to perform "submerged electrodispersion" in insulating liquids which are immiscible to the dispersed phase. ${ }^{13}$ In this work, we attempted to overcome the drawbacks of conventional microfluidic platforms by developing an off-chip method enabled by submerged electrodispersion for the high-throughput production of uniformly sized EGaIn liquid metal microdroplets. We investigated the capabilities of this method for controlling the size of the produced EGaIn droplets and introduced a spinning disk to prevent the coalescence of the produced microdroplets.

Figure 1(a) illustrates the droplet production part of the platform (also see Fig. S1 of the supplementary material for more details). The electrodispersion system comprises a $34 \mathrm{G}$ stainless-steel blunt needle (inner and outer diameters of 60 and $250 \mu \mathrm{m}$, respectively; length of $50 \mathrm{~mm}$ ) charged at few kilovolts and a ground copper electrode positioned few millimeters away. The copper electrode was electrically insulated by a Teflon sleeve. The gap $g$ between the needle and the copper electrode can be adjusted using a guide rail. A DC motor $(12 \mathrm{~V}, 2.1 \mathrm{~kg} / \mathrm{cm}$ at $70 \mathrm{RPM})$ was utilized to drive the tungsten steel shaft (diameter and length of 5 and $100 \mathrm{~mm}$, respectively) via a 3:1 transmission gear set, while a poly(methyl methacrylate) (PMMA) disk (thickness and diameter of 3 and $40 \mathrm{~mm}$, respectively) was fixed
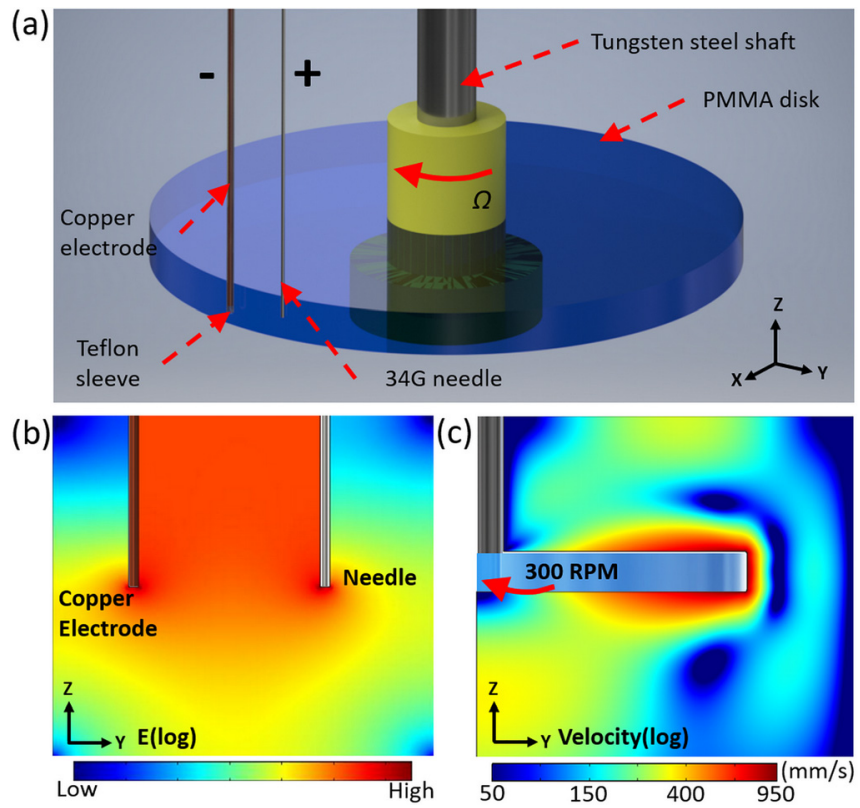

FIG. 1. Experimental setup of the platform for high-throughput and off-chip EGaln microdroplet production. (a) Schematic representation of the droplet generation part of the platform. (b) Color contour of the electric field between the copper electrode and the needle. (c) Velocity profile around a rotating PMMA disk obtained from the numerical simulation. at the end of the shaft. The needle and the ground electrode were placed at the edge of the PMMA disk and did not rotate together with the disk [Figs. 1(a) and S1]. We used a pulse width modulation (PWM) motor controller to adjust the rotational speed $\Omega$ (RPM) of the PMMA disk. The needle and the ground electrode were connected to the positive and negative outputs of a high-voltage DC-DC boost converter, respectively.

We first conducted numerical simulations (COMSOL 5.2 software package, Burlington, MA, USA) for the platform to examine the feasibility of operation. When the distance $g$ is set at $5 \mathrm{~mm}$, the simulated distribution of the electric field for the area between the needle and the electrode ( $y$ - $z$ plane) is presented in Fig. 1(b), from which we can see that the tip of the needle has a localized high-intensity electric field. Figure 1(c) shows the distribution of velocity (perpendicular to the $y$ - $z$ plane) for the liquid surrounding the PMMA disk when $\Omega$ is set at 450 RPM, from which we observed the induction of flow with a high velocity close to the surface of the disk. The purpose of using such a spinning disk is to avoid the coalescence of the produced EGaIn microdroplets on the copper electrode (this phenomenon will be elaborated in detail in the following parts).

In our platform, breakup of emerging liquid metal droplet occurs when the disruptive forces, including drag induced by the flow of oil and the Coulomb force induced by the high voltage, overcome the interfacial tension that resists deformation of the droplet. ${ }^{17}$ Neglecting the effects of the dispersed phase (EGaIn) inertia and assuming that there is no gap between the needle tip and the edge of the disk, by balancing the drag $\left(F_{\text {drag }}\right)$, Coulomb force $\left(F_{E}\right)$, and surface tension induced force $\left(F_{\gamma}\right)$, we can obtain the following polynomial to estimate the diameter of the produced microdroplets $D_{\text {droplet }}$ (see supplementary material S2 for the detailed deduction of this equation):

$$
\left|-\frac{C^{2} V^{2}}{4 \varepsilon g^{2}} D_{\text {droplet }^{5}}+3 C a(1-\beta) \gamma D_{\text {droplet }^{2}}{ }^{2}\right|=\gamma D_{\text {needle }}{ }^{2},
$$

where $C$ is the capacitance per unit area on the emerging EGaIn droplet, $V$ is the applied voltage, $\varepsilon$ is the permittivity of oil, $g$ is the gap between the needle and the ground electrode, $\gamma$ is the interfacial tension between liquid metal and oil, $D_{\text {needle }}$ is the inner diameter of the needle used for the disperse phase, $\beta$ is the ratio between the EGaIn droplet velocity $v_{\text {droplet }}$ and the velocity of the continuous oil phase $v_{c p}$ (i.e., $\beta=v_{\text {droplet }} / v_{c p}$ ) when droplet breakup occurs, and $\mathrm{Ca}$ is the capillary number, which can be defined as

$$
C a=\frac{\eta_{c p} v_{c p}}{\gamma}=\frac{\pi \eta_{c p} \Omega r}{30 \gamma},
$$

where $\eta_{c p}$ is the viscosity of the continuous oil phase, and $r$ is the distance from the needle tip to the axis of rotation of the disk (see Fig. S2 of the supplementary material). The first term on the left-hand side of Eq. (1) represents the Coulomb force $\left(F_{E}\right)$ contributed by the charged droplet upon applying a voltage, and the second term of Eq. (1) represents the drag $\left(F_{\text {drag }}\right)$ exerted on the emerging droplet. Since EGaIn has a high electrical conductivity, the EGaIn-oil interface is charged and behaves as a capacitor when a voltage is applied. The surface tension $\gamma$ between EGaIn and the oil phase depends on the amount of electrical energy stored in the capacitor, which can be described by Lippman's equation: $\gamma=\gamma_{o}-0.5 C V^{2}$, where $\gamma_{o}$ is the maximum surface tension when $V=0$. 
We first conducted a series of experiments to study the effect of high voltage on the EGaIn microdroplet production without rotating the PMMA disk. In this case, no drag will contribute to the break of the droplet $(\Omega=0)$ and Eq. (1) can be simplified to estimate the droplet diameter $D_{\text {droplet }}$ as

$$
D_{\text {droplet }}=\sqrt[5]{\frac{4 \varepsilon g^{2} D_{\text {needle }}{ }^{2}\left(\gamma_{o}-\frac{1}{2} C V^{2}\right)}{C^{2} V^{2}}} .
$$

Equation (3) indicates that increasing the applied voltage $V$ will reduce the surface tension of the emerging EGaIn droplet and meanwhile induce a larger Coulomb force to yield smaller droplets. To verify this, we used a syringe pump (Legato 100, KD Scientific, Holliston, MA, USA) to drive EGaIn liquid metal into the $34 \mathrm{G}$ needle with a flow rate of $50 \mu \mathrm{l} / \mathrm{min}$. The needle and the ground electrode were submerged into a lab beaker filled with sunflower oil. The production of the liquid metal was monitored by a high-speed camera (FPS1000, The Slow Motion Camera Company Ltd., UK) focused on the needle tip. We observed the formation of large droplets (diameter $D$ of $\sim 900 \mu \mathrm{m}$ ) without the application of high voltage since gravity and surface tension are the only forces acting on droplets, as shown in Fig. 2(a). The diameter of the produced EGaIn droplets reduced significantly to $\sim 250 \mu \mathrm{m}$ upon the application of a $600 \mathrm{~V} \mathrm{DC}$ voltage, and the droplet size can be further decreased to $\sim 80 \mu \mathrm{m}$ by increasing the voltage to $2.6 \mathrm{kV}$ [Fig. 2(a)]. The production process of the EGaIn microdroplets at different voltages is also shown in Movie S1. We monitored the electrical current in our experiments and found that the measured current is very low $(<5 \mu \mathrm{A})$ even at the maximum applied voltage of $2.6 \mathrm{kV}$.

Interestingly, unlike the production of water microdroplets, the platform produces EGaIn droplets in the dripping mode without the formation of a Taylor cone even at the highest voltage of $2.6 \mathrm{kV}$. This is evidenced by the sequential snapshots obtained for the production of a single EGaIn microdroplet at $2.6 \mathrm{kV}$ [Fig. 2(b)]. The estimated Weber number ( $W e=\rho D_{\text {needle }} v_{E G a I n}{ }^{2} / \gamma$, where $\rho$ is the EGaIn density, and $v_{E G a I n}$ is the EGaIn dispersed phase velocity within the needle) for EGaIn liquid metal is $\sim 0.05$, which is far less than the value $(\sim 4)$ required for operating in the jetting regime. ${ }^{12}$ Such a small value of We is due to the large surface tension of EGaIn, and this prevents the system from operating in the jetting mode. Figure 2(c) summarizes the diameters of the produced microdroplets with respect to the applied voltage, and we found that this method is able to produce nearmonodisperse EGaIn microdroplets (see Fig. S3 for the characterization of the droplet size distributions).

EGaIn microdroplets can form an oxide layer when exposed to oxygen and the oxide layer normally can prevent the produced droplets from merging. However, since the produced EGaIn microdroplets are positively charged within the oil phase, the strong attractive Coulomb force induced by the negatively charged ground electrode immobilizes the droplets at the Teflon sleeve slightly below the ground electrode to form chains of microdroplets. Such an effect can induce the coalescence of the immobilized microdroplets along the chain, as shown in Fig. 2(d).

To prevent the undesired coalescence of droplets, we introduced a spinning disk $(\Omega=450 \mathrm{RPM})$ to create a flow of oil at the needle tip for dragging the produced microdroplets away from the ground electrode. Interestingly, instead of forming large EGaIn droplets at $0 \mathrm{~V}$, the shear induced by the oil flow [see Fig. 1(c)] at the edge of the (a) $0 \mathrm{k}$

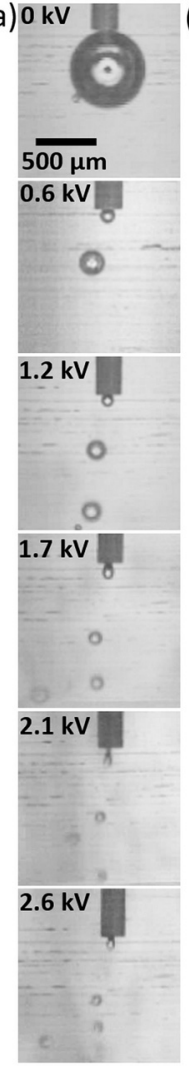

(b) $0 \mathrm{~ms}$
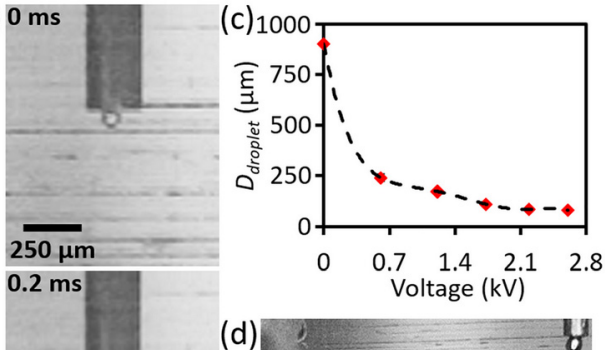

(d)

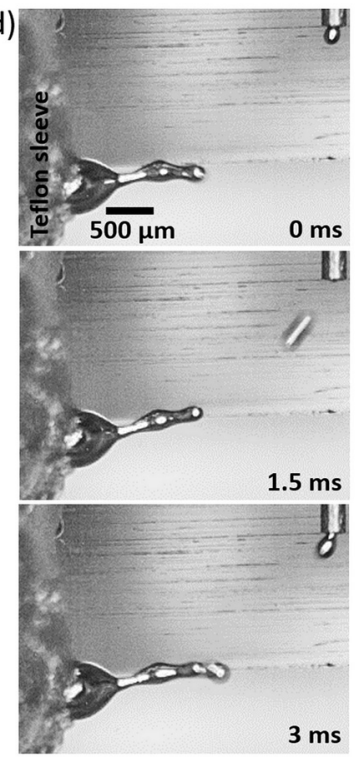

FIG. 2. Controlling the size of the produced EGaln microdroplets using the electric field. (a) Images showing the production of EGaln microdroplets at different voltages without rotating the PMMA disk. (b) Snapshots taken from a high-speed camera showing the formation of an EGaln microdroplet when applying a $2.6 \mathrm{kV}$ DC voltage. (c) Plot of droplet diameter $D$ vs applied voltage corresponding to the micrographs in (a). (d) Sequential snapshots showing the coalescence of the produced EGaln microdroplets $(V=1.7 \mathrm{kV})$.

disk can break the EGaIn phase into microdroplets with a much smaller size (diameter of $\sim 140 \mu \mathrm{m}$ ), as shown in Fig. 3(a). In this case $(V=0)$, droplet production is only due to the fact that drag exerted on the emerging droplet overcomes the interfacial tension of the droplet; as such, Eq. (1) can be simplified to estimate the droplet diameter $D_{\text {droplet }}$ as

$$
D_{\text {droplet }}=\frac{D_{\text {needle }}}{\sqrt{3 C a(1-\beta)}} .
$$

Equation (4) reveals that the droplet diameter resulting from breakup in the dripping mode is a function of $\mathrm{Ca}$. To examine this, we conducted a series of experiments by rotating the PMMA disk at different speeds without applying the DC voltage [formation of a single microdroplet is shown in Fig. S4(a)]. Keeping the flow rate constant at $50 \mu \mathrm{l} /$ min, we observed that increasing $\Omega$ can efficiently reduce the size of the produced EGaIn microdroplets (from $\sim 340 \mu \mathrm{m}$ at $75 \mathrm{RPM}$ to $\sim 140 \mu \mathrm{m}$ at $275 \mathrm{RPM}$ ), as shown in Fig. S4(c). We found that the experimental results are in relatively good agreement with our theoretical prediction [see Fig. S4(c)]. 
(a)

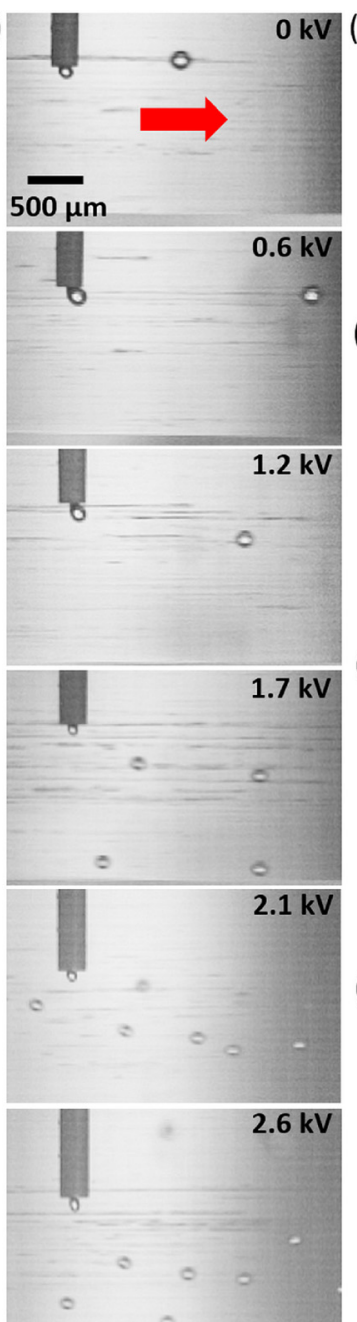

(b)

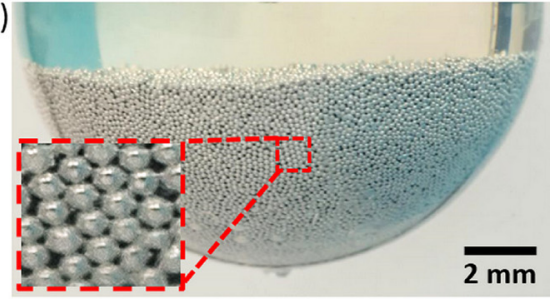

(c) 150

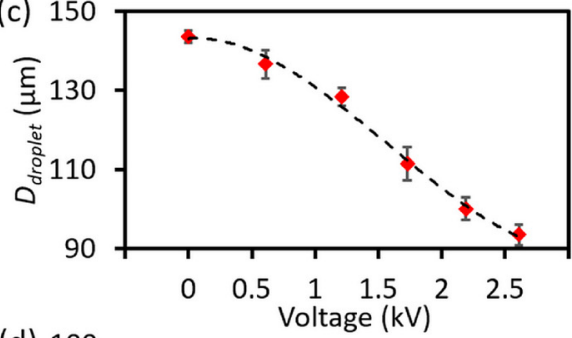

(f)
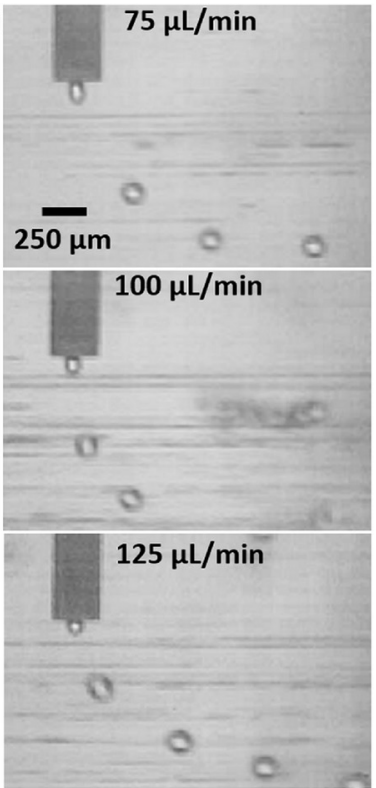

$150 \mu \mathrm{L} / \mathrm{min}$
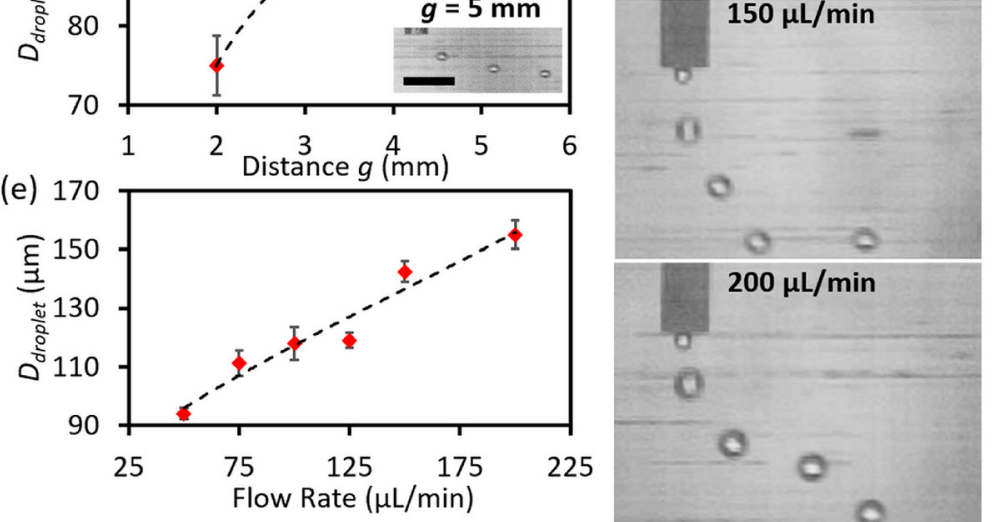

FIG. 3. Mass production of EGaln microdroplets. (a) Images showing the production of EGaln microdroplets at different voltages with a spinning disk ( $\Omega=450$ RPM). (b) Image of the produced EGaln microdroplets (voltage of $1.2 \mathrm{kV}$ ) collected in a test tube. The inset is a magnified image for a few microdroplets. Droplet diameter $D$ vs (c) voltage, (d) gap g, and (e) EGaln flow rate plots for the produced microdroplets. The insets of (d) show the images of the EGaln microdroplet production when $\mathrm{g}$ is set a 2 and $5 \mathrm{~mm}$, respectively, and scale bars are $500 \mu \mathrm{m}$. The value of the error bar is the standard deviation of the droplet diameter. (f) Images showing the production of EGaln microdroplets at different flow rates of EGaln.

When applying a high voltage and meanwhile rotating the PMMA disk, we found that the induced flow at the edge of the disk was able to overcome the attractive Coulomb force and drag the produced microdroplets away from the ground electrode located at the upstream (left-hand side of the needle) even when a high voltage of $2.6 \mathrm{kV}$ is applied [Fig. 3(a)]. The production process of the EGaIn microdroplets at different voltages is also shown in Movie S2. The produced EGaIn microdroplets can quickly sink to the bottom of the beaker due to their high density. This method efficiently prevents the coalescence of droplets at the ground electrode, enabling mass production and collection of the EGaIn microdroplets, as shown in Fig. 3(b).

Figure 3(c) summarizes the diameter of the produced microdroplets with respect to the applied voltage when rotating the disk at 450 RPM (see Fig. S5 for the characterization of the droplet size distributions at different voltages). We found that the produced droplets are near-monodisperse with the coefficient of variation $(\mathrm{CV})$ of the size less than $5 \%$. In comparison to the case without the rotating disk [see Fig. 2(c)], we can see that the shear induced by the spinning disk can result in smaller microdroplets when the applied voltage is less than $1.7 \mathrm{kV}$. However, the platform produces microdroplets with a similar size with or without the spinning disk if the voltage is larger than $1.7 \mathrm{kV}$, indicating that the electrodispersion effect is the dominant factor for reducing the size of the droplets at high voltages. Apart from increasing the voltage, decreasing the gap $g$ can also increase the intensity of the electric field to further reduce the size of the EGaIn microdroplets, as shown in Fig. 3(d). This result is in a good agreement with Eq. (3). Nonetheless, even with the spinning disk, smaller $g$ can induce a larger Coulomb force to chain up the microdroplets and cause 
coalescence. Figures 3(e) and 3(f) illustrate that at a constant voltage of $2.6 \mathrm{kV}$, a higher flow rate of EGaIn can result in the production of droplets with a larger size. The size distributions of the produced droplets at different flow rates are given in Fig. S6. This experiment indicates that the platform is able to produce uniformly sized EGaIn microdroplets in a high-throughput manner, which is impossible to achieve in conventional microfluidic platforms.

In this study, the produced EGaIn droplets have a diameter ranging from tens to hundreds of micrometers, which is comparable with the size of the droplets produced using microfluidic devices. ${ }^{9,10}$ In addition, droplets produced using this platform have a good uniformity $(\mathrm{CV}<$ $5 \%$ ), indicating that our method is also comparable with droplets produced using microfluidic devices. However, the operation of this off-chip platform is much simpler, and the throughput can be significantly improved $(Q>50 \mu \mathrm{l} / \mathrm{min})$. Moreover, our results also show that the size of the produced droplets can be actively tuned by varying the applied voltage, the EGaIn flow rate, and the rotational speed of the disk.

In conclusion, we developed an innovative platform using submerged electrodispersion for the off-chip production of nearmonodisperse $(\mathrm{CV}<5 \%)$ liquid metal microdroplets with the diameter ranging from tens to hundreds of micrometers in a highthroughput manner. We demonstrated that the size of the EGaIn microdroplets can be actively controlled using high voltages, and the introduction of a spinning disk can efficiently avoid the coalescence of the produced EGaIn microdroplets. The excellent capabilities demonstrated for this technique may enable a vast potential for the further development of biomedical, chemical, and electronic applications based on liquid metal microdroplets.

See supplementary material for additional schematics of the experimental setup, deduction of Eqs. (1)-(4), prediction of the droplet size, and characterization of droplet size distribution.

Dr. Shi-Yang Tang is the recipient of the Vice-Chancellor's Postdoctoral Research Fellowship funded by the University of
Wollongong. Professor Weihua Li acknowledges the ARC Discovery Grant support (Grant No. DP180100055).

\section{REFERENCES}

${ }^{1}$ W. Zhang, J. Z. Ou, S.-Y. Tang, V. Sivan, D. D. Yao, K. Latham, K. Khoshmanesh, A. Mitchell, A. P. O’Mullane, and K. Kalantar-zadeh, Adv. Funct. Mater. 24(24), 3799 (2014).

${ }^{2}$ S.-Y. Tang, B. Ayan, N. Nama, Y. Bian, J. P. Lata, X. Guo, and T. J. Huang, Small 12(28), 3861 (2016).

${ }^{3}$ W. Zhang, B. S. Naidu, J. Z. Ou, A. P. O’Mullane, A. F. Chrimes, B. J. Carey, Y. Wang, S.-Y. Tang, V. Sivan, A. Mitchell, S. K. Bhargava, and K. Kalantarzadeh, ACS Appl. Mater. Interfaces 7(3), 1943 (2015).

${ }^{4}$ C. Ladd, J.-H. So, J. Muth, and M. D. Dickey, Adv. Mater. 25(36), 5081 (2013); S.-Y. Tang, J. Zhu, V. Sivan, B. Gol, R. Soffe, W. Zhang, A. Mitchell, and K. Khoshmanesh, Adv. Funct. Mater. 25(28), 4445 (2015).

${ }^{5}$ Y. Yu and E. Miyako, iScience 3, 134 (2018).

${ }^{6}$ C. Guo, Y. Yu, and J. Liu, J. Mater. Chem. B 2(35), 5739 (2014).

${ }^{7}$ E. J. Markvicka, M. D. Bartlett, X. Huang, and C. Majidi, Nat. Mater. 17(7), 618 (2018); N. Kazem, T. Hellebrekers, and C. Majidi, Adv. Mater. 29(27), 1605985 (2017); G. Yun, S.-Y. Tang, S. Sun, D. Yuan, Q. Zhao, L. Deng, S. Yan, H. Du, M. D. Dickey, and W. Li, Nat. Commun. 10, 1300 (2019).

${ }^{8}$ M. D. Dickey, ACS Appl. Mater. Interfaces 6(21), 18369 (2014).

${ }^{9}$ J. Thelen, M. D. Dickey, and T. Ward, Lab Chip 12(20), 3961 (2012); T. Hutter, W.-A. C. Bauer, S. R. Elliott, and W. T. S. Huck, Adv. Funct. Mater. 22(12), 2624 (2012).

${ }^{10}$ S.-Y. Tang, I. D. Joshipura, Y. Lin, K. Kalantar-Zadeh, A. Mitchell, K. Khoshmanesh, and M. D. Dickey, Adv. Mater. 28(4), 604 (2016).

${ }^{11}$ K. Khoshmanesh, S.-Y. Tang, J. Y. Zhu, S. Schaefer, A. Mitchell, K. Kalantarzadeh, and M. D. Dickey, Lab Chip 17(6), 974 (2017).

${ }^{12}$ L. L. F. Agostinho, C. U. Yurteri, E. C. Fuchs, and J. C. M. Marijnissen, Appl. Phys. Lett. 100(24), 244105 (2012).

${ }^{13}$ C. J. Young, L. A. Poole-Warren, and P. J. Martens, Biotechnol. Bioeng. 109(6), 1561 (2012).

${ }^{14}$ C. Larriba-Andaluz and J. F. de la Mora, Phys. Fluids 22(7), 072002 (2010).

${ }^{15}$ M. A. Henderson and J. S. J. C. C. McIndoe, Chem. Commun. 2006(27), 2872; A. M. Gañán-Calvo and J. M. J. P. R. E. Montanero, Phys. Rev. E 79(6), 066305 (2009).

${ }^{16}$ F. J. Higuera, Phys. Fluids 22(11), 112107 (2010).

${ }^{17}$ J. Husny and J. J. Cooper-White, J. Non-Newtonian Fluid Mech. 137(1), 121 (2006). 